Apparently no side-reaction occurred, such as urea formation, because the amine products, which were solid, melted sharply within two degrees of the reported melting points $^{7}$ without recrystallization. Those amines which were liquids were also uncontaminated because their infra-red curves ${ }^{8}$ were identical to original samples.

It appears that urea formation is decreased to a considerable degree when the Schmidt reaction is run at room temperatures. Furthermore, the increase of yields in polyphosphoric acid over conventional methods seems to imply the formation of the more reactive oxocarbonium ion in this medium.

Richard F. Stockei*

David M. HaLI $\dagger$

Department of Chemistry and Geology

and the Department of Textile Chemistry,

Clemson College, Clemson, South Carolina.

* Present address: American Cyanamid Co., Research and Development, Bound Brook, New Jersey.

$\dagger$ Present address: College of Science and Technology, University of Tanchester.

'Snyder, H. R., and Elstron, C. T., J. Amer. Chem. Soc., 76, 3039 (1954).

${ }^{2}$ Conley, R. T., Chem. and Indust., 438 (1958).

${ }^{3}$ Conley, R. T., and Nowok, B. E., Chem, and Indust., 1161 (1956).

' Snyder, H. R., Elston, C. T., and Kellom, D. B., J. Amer. Chem. Soc. 75, 2014 (1953).

s Snyder, H. R., and Uhlig, F., in Advances in Organic Chemistry, 1, 69 (Interscience Publishers, Inc., New York, 1960).

- Briggs, L. H., and Lyttleton, J. W., J. Chem. Soc., 421 (1943).

'Shriner, R. L., Fuson, R. C., and Curtin, D. Y., The Systematic Identification of Organic Compounds, fourth ed. (John Wiley and Sons, Inc. New York).

* Bellamy, L. J., The Infra-red Spectra of Complex Molecules, second ed. 249 (John Wiley and Sons, Inc., New York)

\section{Effect of Naphthalene on the Quenching of Liquid Scintillation Solutions}

Is liquid scintillation counting the passage of $\beta$-particles through the phosphor solution results in a complex series of events, the useful ones culminating in the emission of photoelectrons at the photocathode of the photomulti. plior. A number of parasitic events occur concomitantly that lead to a lowering of the yield, or total loss of the photoelectrons, that is, quenching. Possible sequences for these events, which occur during a time-interval of approximately $10^{-9} \mathrm{sec}$, have been outlined by several authors ${ }^{1-3}$. The addition of naphthalene to some liquid phosphors improves their counting characteristics. The enhancement in counting efficiency has been ascribed by Fust and Kallmann ${ }^{4}$ to the ability of the naphthalene to act as an intermediate solvent that is capable of transmitting the energy from the primary solvent to the scintillators, thereby by-passing the quenching agent. If this is true the series of events leading to photoelectron production could be outlined as follows: $(A)$ emission of $\beta$-particle; $(B)$ excitation of primary solvent molecules (toluene); $(C)$ excitation of secondary solvent molecules (naphtha. lene); $(D)$ excitation of primary solute molecules (PPO); $(E)$ excitation of secondary solute molecules (POPOP); $(F)$ production of photons.

I have found that the addition of naphthalene to some liquid scintillator systems containing tritium can have a marked effect on the quenching curves. The effect depends entirely on the chemical nature of the quenching agent. With some substances the quenching effect is almost entirely removed; with others, it is enhanced.

Tho continuous curves shown in Figs. 1 and 2 were prepared by adding inactive benzoic acid and methyl benzoate to a liquid scintillator $(4 \mathrm{~g} / \mathrm{l}$. PPO and 0.04 $\mathrm{g} / \mathrm{l}$. POPOP in toluene) containing approximately $10^{-8} \mathrm{~g}$ of tritiated benzoic acid. The broken curves were prepared by repeating the experiments with the same liquid phosphor solutions containing in addition $100 \mathrm{~g} / \mathrm{l}$. of purified naphthalene.

It can be seen from the results that addition of naphthalene to the liquid scintillator containing benzoic acid

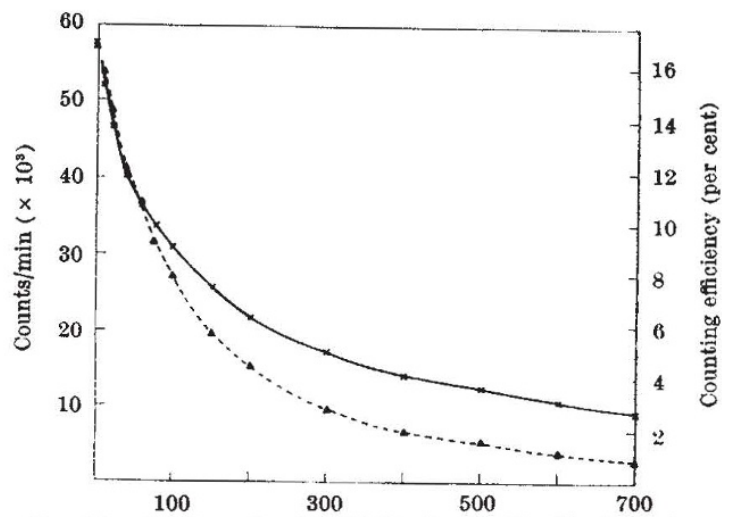

$\mathrm{Mg}$ of inactive benzoic acid added to $9 \mathrm{ml}$. of liquid scintillator Fig. 1. Graph showing the effect on the count-rate of adding inactive benzoic acid to liquid scintillators containing a very small quantity of tritiated benzoic acid. $\times$, Normal scintillator solution; $A$, scin-

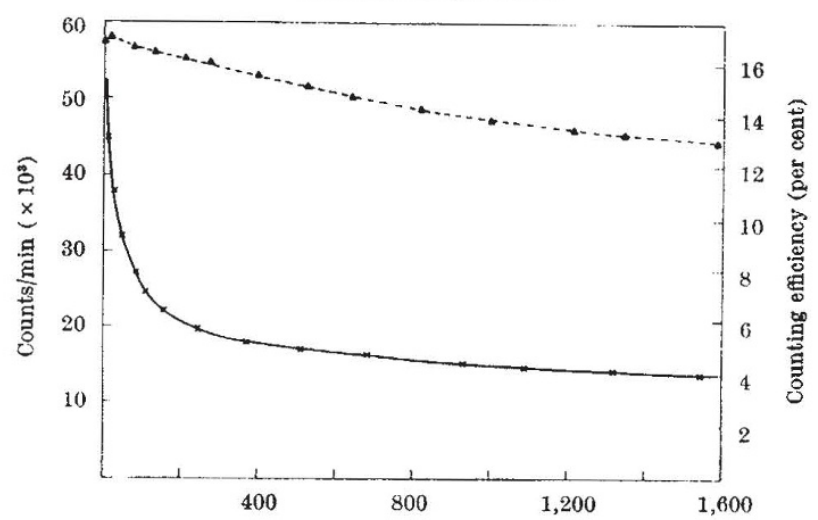

$\mathrm{Mg}$ of inactive methyl benzoate added to $9 \mathrm{ml}$. of liquid scintillator Fig. 2. Graph showing the effect of adding naphthalene to the scintillator on the tritium-methyl benzoate quenching curve. The small increase in count-rate recorded on the broken curve for very small amounts of methyl benzoate is due to desorption of active tritiatod scintillator; $A$, scintillator plus naphthalene

results in a small increase in counting efficiency at low concentrations of benzoic acid, and a decrease in efficiency at high concentrations. The addition of naphthalene to a benzoate-quenched solution, on the other hand, results in is considerable increase in counting efficiency at all quencher concentrations.

These results would seem to indicate that the quenching effects of benzoic acid and methyl benzoate are basically different. Two possible explanations are: $(a)$ that the quenching phenomena for the two quenching agents occur at different points in the chain of events outlined here; or $(b)$ that the quenching phenomena operate at the same stage in the scintillation process (between events $B$ and $D$ ) and are the result of different rates of energy transfer. Thus, if quenching occurs between events $B$ and $C$ the rate of transfer of energy from the primary solvent to benzoic acid is greater than its rate of transfer to naphthalene, which in turn is greater than the transfer rate to methyl benzoste. If, however, quenching occurs between events $C$ and $D$, the rates of energy transfer from naphthalene would be in the order benzoic acid $>$ PPO $>$ methyl benzoate.

Horace E. Dobes

U.K. Atomic Energy Authority, Isotope Research Division,

Wantage Research Laboratory, Wantage, Berks.

${ }^{1}$ Hayes, F. N., J. App. Rad. and Isot., 1, 46 (1956).

3 Birks, J. B., Proc. Inst. Rad. Eng. Seventh Scintillation Counter Symp., Washington, 1 (1960)

${ }^{8}$ Funt, B. L., and Hetherington, A., J. App. Rad. and 18ot., 13, 215 (1962).

4Fust, M. and Kallmann, H., Phys. Rev. 97,583 (1955).

$\checkmark$ Dobbs, H. E., U.K.A.E.A. Publ. AERE M1075 (1962). 